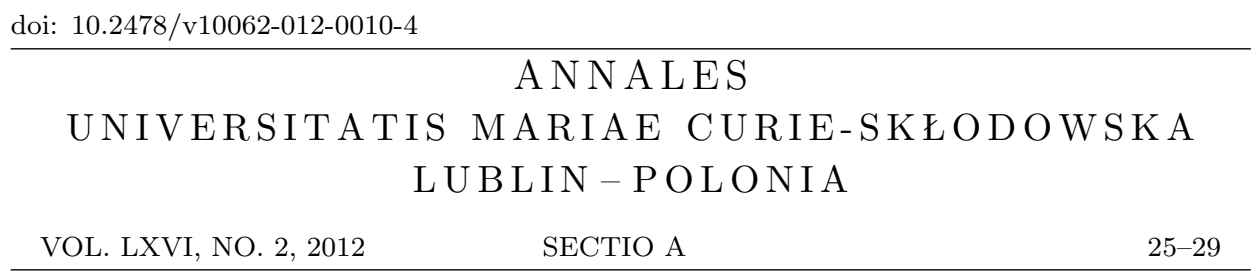

DAOUD BSHOUTY and ABDALLAH LYZZAIK

\title{
On a question of T. Sheil-Small regarding valency of harmonic maps
}

Dedicated to Bogdan Bojarski on the occasion of his 80th birthday

\begin{abstract}
The aim of this work is to answer positively a more general question than the following which is due to T. Sheil-Small: Does the harmonic extension in the open unit disc of a mapping $f$ from the unit circle into itself of the form $f\left(e^{i t}\right)=e^{i \varphi(t)}, 0 \leq t \leq 2 \pi$, where $\varphi$ is a continuously non-decreasing function that satisfies $\varphi(2 \pi)-\varphi(0)=2 N \pi$, assume every value finitely many times in the disc?
\end{abstract}

Introduction. Let $\mathbb{D}$ and $\mathbb{T}$ be the open unit disc and the unit circle respectively, and let $N$ be a positive integer. An $\boldsymbol{N}$-valent quasi-homeomorphism from the unit circle into itself is a circle mapping $f: \mathbb{T} \rightarrow \mathbb{T}$ of the form $f\left(e^{i t}\right)=e^{i \varphi(t)}, 0 \leq t \leq 2 \pi$, where $\varphi$ is a non-decreasing function that satisfies $\varphi(2 \pi)-\varphi(0)=2 N \pi$. It can be seen that every such quasi-homeomorphism is a pointwise limit of a sequence of circle mappings $f_{n}: \mathbb{T} \rightarrow \mathbb{T}$ of the form $f_{n}\left(e^{i t}\right)=e^{i \varphi_{n}(t)}, 0 \leq t \leq 2 \pi$, where $\varphi_{n}$ is a continuously strictly increasing function that satisfies $\varphi_{n}(2 \pi)-\varphi_{n}(0)=2 N \pi$.

A 1-valent quasi-homeomorphism is referred to as quasi-homeomorphism.

The celebrated Radó-Kneser-Choquet Theorem can be stated as follows.

2000 Mathematics Subject Classification. Primary 26C10; Secondary 30C15.

Key words and phrases. Harmonic mapping, cluster set. 
Theorem A (Radó-Kneser-Choquet Theorem [3, pp. 29-34]). Suppose that $F$ is the harmonic extension in $\mathbb{D}$ of a quasi-homeomorphism $f$ from the unit circle into itself. Then $F$ is univalent.

In an attempt to generalize this theorem to 2-valent quasi-homeomorphisms $f$ from the unit circle into itself, it was suggested that the respective functions $F$ are at most 4-valent. However, examples presented in [2] have shown that some of these mappings could be 6 -valent or 8 -valent. Furthermore, the construction procedure used in the paper suggested the possibility of finding a 2-valent quasi-homeomorphism from the unit circle into itself whose harmonic extension in $\mathbb{D}$ assumes a predetermined finite valency. But this remains an open problem.

In a personal communication with the first author about a decade ago, T. Sheil-Small raised the following question:

If $F$ is the harmonic extension in $\mathbb{D}$ of a mapping $f$ of the form $f\left(e^{i t}\right)=e^{i \varphi(t)}, 0 \leq t \leq 2 \pi$, where $\varphi$ is a continuously non-decreasing function that satisfies $\varphi(2 \pi)-\varphi(0)=2 N \pi$, then does $F$ assume every value finitely many times in the disc?

In this note, we show that the answer to this question is positive. In fact, a more general result is shown henceforth to be true.

For a function $F: \mathbb{D} \rightarrow \mathbb{C}$ and a point $\zeta \in \mathbb{T}$, let $C(F, \zeta)$ and $C(F, \mathbb{T})$ denote the cluster sets of $F$ at $\zeta$ and on $\mathbb{T}$ respectively.

The result of the note can be stated as follows.

Theorem 1. Suppose that $F$ is the harmonic extension in $\mathbb{D}$ of an $N$-valent quasi-homeomorphism $f$ from the unit circle into itself that takes on three distinct values. Then $F$ takes on every point in $\mathbb{D} \backslash C(F, \mathbb{T})$ finitely many times.

As a consequence we have:

Corollary 2. Suppose that $F$ is the harmonic extension in the open unit disc of a mapping $f$ of the form $f\left(e^{i t}\right)=e^{i \varphi(t)}, 0 \leq t \leq 2 \pi$, where $\varphi$ is a continuously non-decreasing function that satisfies $\varphi(2 \pi)-\varphi(0)=2 N \pi$. Then $F$ takes on every point in $\mathbb{D}$ finitely many times.

Before embarking on the proof of Theorem 1, we define an algebraic curve as a connected component of the preimage of a straight line or circle under an analytic function.

Proof of Theorem 1. Write $F=u+i v$, where $u$ and $v$ are the real and imaginary parts of $F$. Suppose that there exist a point $\omega \in \mathbb{D} \backslash C(F, \mathbb{T})$ and a set $S$ of countably infinitely many distinct values $z_{n} \in \mathbb{D}, n=1,2, \ldots$ such that $F\left(z_{n}\right)=\omega$ for all $n$; note that $\left|z_{n}\right| \leq \rho<1$ for some $\rho$ since $\omega \notin C(F, \mathbb{T})$. Let $\omega=u_{0}+i v_{0}$ for $u_{0}, v_{0} \in \mathbb{R}$; then $u\left(z_{n}\right)=u_{0}$ and $v\left(z_{n}\right)=v_{0}$ for all $n$. 
Consider the level set $u=u_{0}$; note that this is a set-union of mutually disjoint algebraic curves. Suppose that each of these curves carries a finite subset of $S$ of the points $z_{n}$. Then these curves are countably infinite and may be denoted by $C_{n}, n=1,2, \ldots$. Label one of the points of $S \cap C_{n}$ by $\zeta_{n}$ for every $n$. Since $\left|\zeta_{n}\right| \leq \rho<1$ for all $n$, there exists a subsequence $\left(\zeta_{n_{k}}\right)$ of $\left(\zeta_{n}\right)$ that converges to a point $\zeta$. Evidently, $|\zeta| \leq \rho<1, F(\zeta)=\omega$ and $\zeta$ belongs to some level curve $C: u=u_{0}$. This yields a contradiction since near $\zeta$ the curve $C$ fails to be isolated from the level curves $C_{n}$.

It follows that the level set $u=u_{0}$ is a disjoint union of finitely many algebraic curves of which one, say $C$, carries countably infinitely many points $z_{n}$ that we denote by $\zeta_{1}, \zeta_{2}, \ldots$ Observe the following:

(1) $C$ never encloses a Jordan domain in $\mathbb{D}$ because of the maximum principle for harmonic functions;

(2) $C$ is a union of analytic Jordan arcs $\gamma$ that are mutually disjoint except possibly for a common critical point of $u$;

(3) Every $\gamma$ clusters in $\mathbb{T}$.

Suppose that some arc $\gamma$ accumulates on a non-degenerate subarc $J \subset \mathbb{T}$; denote the interior of $J$ by $J^{\circ}$. Let $\eta \in J^{\circ}$. Note that in every direction towards $\eta$ from $\mathbb{D}$ there exists a sequence of points in $\gamma$ converging to $\eta$ on which $u$ attains the value $u_{0}$. This entails by a result of Schwarz [1, Theorem 23] that $u$ is continuous and is identically $u_{0}$ on $J^{\circ}$.

Let $g$ be the analytic completion on $u$. By the reflection principle, $g$ is analytic on $J^{\circ}$. Fix $\eta \in J^{\circ}$. It is immediate that $g([0, \eta])$ is an arc that meets the vertical line $L: u=u_{0}$ in the $(u, v)$-plane at countably infinitely many points that are away from infinity. Since both $\operatorname{arcs} g([0, \eta])$ and $L$ are analytic, $g([0, \eta]) \subset L$, see [4, Theorem 7.19, pp. 241-244], and equivalently $u=u_{0}$ on $[0, \eta]$. But $\eta$ is an arbitrary point of $J^{\circ}$; hence $u$ is identically $u_{0}$ on the open circular sector with vertex at the origin and subtending $J^{\circ}$ and consequently on $\mathbb{D}$, which yields a contradiction.

Thus every Jordan arc $\gamma$ terminates in every direction at a point in $\mathbb{T}$. We contend that every $\gamma$ is a crosscut of $\mathbb{D}$. For suppose otherwise, then some $\gamma$ is a loop with a unique point $\eta \in \bar{\gamma} \cap \mathbb{T}$. If $G \subset \mathbb{D}$ is the bounded region enclosed by $\gamma$, then, because $u$ is a bounded harmonic function, the limit

$$
\lim _{z \rightarrow \eta} u(z)=u_{0} \text { through values } z \in \bar{G} .
$$

We infer, by the maximum principle, that $u$ is identically $u_{0}$ in $G$ and consequently in $\mathbb{D}$, which gives a contradiction. This proves our claim.

Suppose now that $\gamma$ terminates at two distinct points $\alpha, \beta \in \mathbb{T}$, and let $\gamma^{\prime} \subset C$ be a crosscut of $\mathbb{D}$ similar to $\gamma$. It is immediate that $\gamma^{\prime}$ can not terminate at both $\alpha, \beta$. In fact, $\gamma^{\prime}$ can neither terminate at $\alpha$ nor at $\beta$. For suppose $\gamma^{\prime}$ terminates at $\alpha$; then, since $C$ is connected, there exists a 
continuum that meets both $\gamma$ and $\gamma^{\prime}$. But then $K \cup \gamma \cup \gamma^{\prime}$ bounds a Jordan subdomain $K$ of $\mathbb{D}$, which gives a contradiction.

It follows at once that $\bar{\gamma}$ and $\overline{\gamma^{\prime}}$ are either disjoint or cross at a singleton in $\mathbb{D}$; namely a critical point of $u$. Thus $\bar{C}$ is a tree whose vertices are the critical points of $u$ and the terminal points of the $\operatorname{arcs} \gamma$. We show that this tree is finite. Suppose otherwise, then the crosscuts $\gamma$ comprising $C$ are countably infinite, and consequently the same are the endpoints of $C$. The latter points subdivide $\mathbb{T}$ into countably infinitely many subarcs $\lambda$. Let $\lambda_{1}$ and $\lambda_{2}$ be two of these arcs that share a common terminal point $\nu$, and let $G_{1}$ and $G_{2}$ be the Jordan domains bounded by $\bar{C} \cup \lambda_{1}$ and $\bar{C} \cup \lambda_{2}$ respectively. Note that $G_{1}$ and $G_{2}$ have a common boundary arc, denoted by $\delta \subset C$, with an endpoint at $\nu$. Evidently, $g(\delta)$ is a line segment of the vertical line $L: u=u_{0}$. Note that $g$, like $u$, has no critical points in the interior of $\delta$ since $g$ and $u$ share these points, and that $u(z) \neq u_{0}$ and $u\left(z^{\prime}\right) \neq u_{0}$ for all $z \in G_{1}$ and $z \in G_{2}$ or else $C \cap\left(G_{1} \cup G_{2}\right)$ is nonempty.

It follows that $g\left(G_{1}\right)$ and $g\left(G_{2}\right)$ lie on different sides of $L$. But by the hypotheses on $f, u-u_{0}$ cannot change the sign more than $N$ times. This implies at once that the number of $\operatorname{arcs} \lambda$ is at most $2 N$; thus the number of crosscuts $\gamma$ comprising $C$ is at most $N$.

We conclude that some crosscut $\gamma$, denoted by $\Gamma$, contains infinitely countably many points $\zeta_{n}$. We may assume without loss of generality that $\zeta_{n} \in \Gamma$ for all $n=1,2, \ldots$.

On the other hand, by undergoing the same discussion on $v$ instead of $u$ we conclude that there exists a crosscut $\Gamma^{\prime}$ that is contained in the level set $v=v\left(z_{0}\right)$ and contains infinitely countably many of the points $\zeta_{n} \in \Gamma, n=$ $1,2, \ldots$. Since every $\left|\zeta_{n}\right| \leq \rho<1 n=1,2, \ldots$ and the $\operatorname{arcs} \Gamma$ and $\Gamma^{\prime}$ are analytic, $\Gamma$ and $\Gamma^{\prime}$ coincide.

Suppose now that $\xi \in \mathbb{T}$ is a terminal point of $\Gamma\left(\right.$ or $\left.\Gamma^{\prime}\right)$. Then

$$
u(z) \rightarrow u_{0} \quad \text { for } z \in \Gamma \quad z \rightarrow \xi
$$

hence $u_{0} \in C(u, \xi)$. By the same token we conclude that $v_{0} \in C(v, \xi)$. Therefore, $\omega \in C(F, \mathbb{T})$ and we have a contradiction to our original assumption. This completes the proof of Theorem 1.

\section{REFERENCES}

[1] Ahlfors, L., Complex Analysis, Third Edition, McGraw-Hill, New York, 1979.

[2] Bshouty, D., Hengartner, W., Lyzzaik, A. and Weitsman, A., Valency of harmonic mappings onto bounded convex domains, Comput. Methods Funct. Theory 1 (2001), 479-499.

[3] Duren, P., Harmonic Mappings in the Plane, Cambridge University Press, Cambridge, 2004.

[4] Markushevich, A. I., Theory of functions of a complex variable. vol. III, English edition translated and edited by Richard A. Silverman, Prentice-Hall Inc., N. J., 1967. 
Daoud Bshouty

Department of Mathematics

Technion

Haifa

Israel

e-mail: daoud@tx.technion.ac.il

Received August 24, 2011
Abdallah Lyzzaik

Department of Mathematics

American University of Beirut

Beirut

Lebanon

e-mail: lyzzaik@aub.edu.1b 